\title{
Numerical simulation of a bubble rising in shear-thinning fluids
}

\author{
Li Zhang a,b, Chao Yang ${ }^{a}$, Zai-Sha Mao ${ }^{\mathrm{a}, *}$ \\ ${ }^{a}$ Key Laboratory of Green Process and Engineering, Institute of Process Engineering, Chinese Academy of Sciences, Beijing 100190, China \\ b State Key Laboratory of Nonlinear Mechanics, Institute of Mechanics, Chinese Academy of Sciences, Beijing 100080, China
}

\section{A R T I C L E I N F O}

\section{Article history:}

Received 19 September 2008

Received in revised form 29 January 2010

Accepted 13 February 2010

\section{Keywords:}

Numerical simulation

Bubble

Level set

Non-Newtonian liquid

\begin{abstract}
A B S T R A C T
The motion of a single bubble rising freely in quiescent non-Newtonian viscous fluids was investigated experimentally and computationally. The non-Newtonian effects in the flow of viscous inelastic fluids are modeled by the Carreau rheological model. An improved level set approach for computing the incompressible two-phase flow with deformable free interface is used. The control volume formulation with the SIMPLEC algorithm incorporated is used to solve the governing equations on a staggered Eulerian grid. The simulation results demonstrate that the algorithm is robust for shear-thinning liquids with large density ( $\rho_{1} / \rho_{\mathrm{g}}$ up to $\left.10^{3}\right)$ and high viscosity $\left(\eta_{1} / \eta_{\mathrm{g}}\right.$ up to $\left.10^{4}\right)$. The comparison of the experimental measurements of terminal bubble shape and velocity with the computational results is satisfactory. It is shown that the local change in viscosity around a bubble greatly depends on the bubble shape and the zero-shear viscosity of non-Newtonian shear-thinning liquids. The shear-rate distribution and velocity fields are used to elucidate the formation of a region of large viscosity at the rear of a bubble as a result of the rather stagnant flow behind the bubble. The numerical results provide the basis for further investigations, such as the numerical simulation of viscoelastic fluids.
\end{abstract}

(c) 2010 Elsevier B.V. All rights reserved.

\section{Introduction}

In diverse gas-liquid systems the gas is frequently dispersed as bubbles in the liquid by means of a sieve plate or a perforated plate such as in bubble column reactors, fermentation, mineral processing, petrochemical processes. It is highly important to obtain the knowledge of bubble behavior not only in a Newtonian continuous phase but also in a non-Newtonian one, because the investigation on bubble motion provides useful and essential information for realizing suitable process design and operation. It is well known that a large number of investigations concerning various aspects of the bubble or drop motion in non-Newtonian fluids have been reported theoretically and experimentally in the past. Many of them have been well summarized and reviewed by Chhabra [1,2] and Kulkarni and Joshi [3]. Nevertheless, in comparison to the study of the bubble or drop motion in Newtonian fluids, various unanswered questions and problems still remain to be considered in non-Newtonian fluid systems due to the inherently complex nature of the non-Newtonian fluids. The above-mentioned studies are mostly based on experiments.

Numerical analysis has become a powerful tool for comprehending and revealing the details of flow structure and mechanism, because some essential physical information peculiar to non-

\footnotetext{
* Corresponding author. Tel.: +86 10 62554558; fax: +86 1062561822.

E-mail addresses: chaoyang@home.ipe.ac.cn (C. Yang), zsmao@home.ipe.ac.cn (Z.-S. Mao).
}

Newtonian fluids, such as the local effect of shear-thinning on bubble motion and mass transfer, can be locally obtained and evaluated by numerical techniques, but hard to be retrieved from experiments. The knowledge of shear-rate and the resulting stress distribution in aerated non-Newtonian liquids are important for determining whether a bioreactor is suitable to handle shearsensitive biosystems [4]. In the recent literature, few attempts have so far been made to analyze numerically the drop or bubble behavior in non-Newtonian fluids. Kishore et al. [5] carried out the numerical investigation to obtain the steady state drag coefficients and flow patterns of a single Newtonian fluid sphere sedimenting in power-law liquids using a finite difference method based SMAC implicit solver on a staggered grid and proposed a simple correlation for the total drag coefficient, which can be used to predict the rate of sedimentation of fluid sphere in power-law liquids. Tsamopoulos et al. [6] simulated the rise of a bubble in a Newtonian or a viscoplastic fluid for a wide range of material parameters based on the mixed finite element method coupled with a quasi-elliptic mesh generation scheme in order to follow the large deformation of the physical domain. Wagner et al. [7] developed a new lattice Boltzmann method to simulate a bubble rising in a viscoelastic fluid with constant viscosity. Pillapakkam and Singh [8] conducted a numerical investigation of bubble and drop behavior in viscoelastic flows with constant viscosity using the level set method. Ohta et al. $[9,10]$ explored both experimentally and numerically the motion of a Newtonian, spherical and deformable drop rising in shearthinning fluids using the VOF method. Radl et al. [11,12] carried out the numerical analysis on the motion of deformed bubbles in non- 


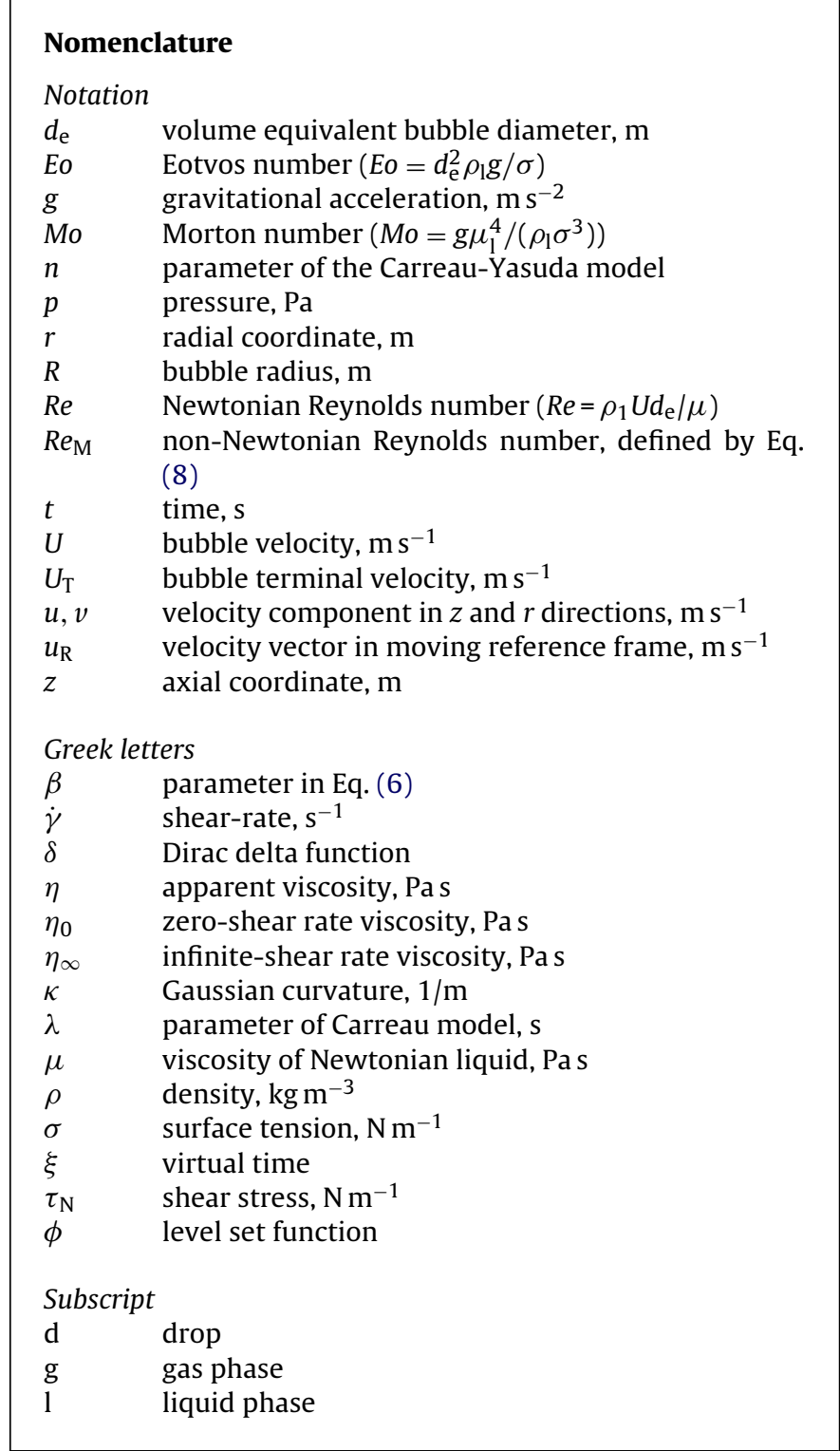

Newtonian fluids and associated mass transfer using first principle methods. As the stability of numerical schemes depends strongly on the ratio between liquid and gaseous phase densities and viscosities, the above investigations $[7,8,11,12]$ represent the bubble using a Newtonian drop of low density. That is, the density and viscosity ratios of liquid over gas phase were up only to 10 , which was much lower than that in real cases $\left(\rho_{\mathrm{l}} / \rho_{\mathrm{g}} \sim 10^{3}\right.$ and $\left.\eta_{\mathrm{l}} / \eta_{\mathrm{g}} \sim 10^{4}\right)$.

In this study, the motion of a single bubble rising freely through generalized Newtonian fluids is computed numerically using a level set method for tracking the bubble interface. The changes in the local viscosity field around a bubble rising in shear-thinning nonNewtonian fluids such as Carreau fluids are examined. Real density and viscosity ratios of gas and liquid are used to describe the shearthinning non-Newtonian two-phase flow systems.

\section{Mathematical models and simulation methods}

The motion of a single bubble rising driven by buoyancy in an immiscible quiescent liquid is considered with the following assumptions: (1) the fluids in both phases are incompressible; (2) the two-phase flow is axisymmetric and laminar; (3) the two-phase flow is isothermal. The computational domain is sketched in Fig. 1.

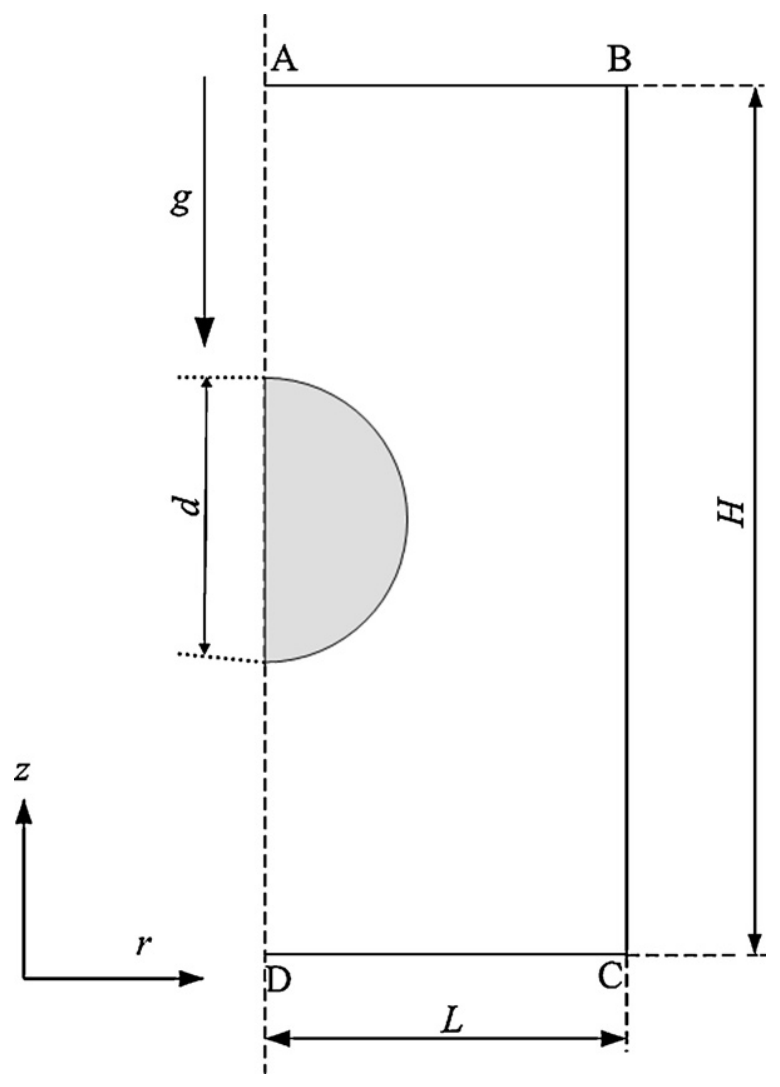

Fig. 1. Schematic diagram of solution domain for numerical simulation.

To prevent the rising bubble from escaping out of the computational domain, the computational domain extends upstream and cuts short downstream the bubble with the same bubble velocity.

\subsection{Governing equations}

In the level set formulation, the transient motion of the bubble can be expressed by the continuity and Navier-Stokes equations as follows:

$\nabla \cdot \boldsymbol{u}=0$

$\rho\left(\frac{\partial \boldsymbol{u}}{\partial t}+\boldsymbol{u} \cdot \nabla \boldsymbol{u}\right)=-\nabla P+\rho \mathbf{g}+\nabla \cdot \tau+\sigma \kappa \delta(\phi) \boldsymbol{n}$

where $\mathbf{u}$ is the velocity vector, $P$ the pressure, $\rho$ the density, $\sigma$ is surface tension, $\mathbf{g}$ is the gravity and $\tau$ the stress tensor. For Newtonian fluids the stress tensor is $\tau=2 \mu \mathbf{D}$, and the strain rate tensor is

$\boldsymbol{D}=\frac{1}{2}\left(\nabla \boldsymbol{u}+\nabla \boldsymbol{u}^{\mathrm{T}}\right)$

\subsection{Constitutive equation for continuous phase}

The stress tensor for a generalized non-Newtonian inelastic fluid is expressed as

$\tau=2 \eta(\dot{\gamma}) D$

where the apparent viscosity $\eta(\dot{\gamma})$ is dependent on the shearrate and the rate-of-strain is:

$\dot{\gamma}=\sqrt{2 \boldsymbol{D}: \boldsymbol{D}}$

Once the local shear-rate is calculated at each nodal point in the computational domain, the field of $\dot{\gamma}$ can be reconstructed. 
The constitutive equation of a purely viscous non-Newtonian fluid can be expressed by the Carreau-Yasuda shear-thinning model [13]:

$\eta(\dot{\gamma})=\eta_{\infty}+\left(\eta_{0}-\eta_{\infty}\right)\left[1+(\lambda \dot{\gamma})^{\beta}\right]^{(n-1) / \beta}$

where $\eta_{0}$ and $\eta_{\infty}$ are the viscosities corresponding to the zero shear-rate and the infinite-shear rate, respectively, $\lambda$ is the inelastic time constant, $n$ is the power-law index, and $\beta$ is a dimensionless parameter describing the transition region between the zero shearrate region and power-law region. In practice, $\beta$ is close to 2 and $\eta_{\infty}$ is small, and therefore Eq. (6) reduces to

$\eta(\dot{\gamma})=\eta_{0}\left[1+(\lambda \dot{\gamma})^{2}\right]^{(n-1) / 2}$

Note that a Newtonian fluid can be recovered as a special case of the present Carreau fluid by letting $n=1$ and/or $\lambda=0$, and a powerlaw fluid can be obtained by assuming a large $\lambda$.

Since the fluid viscosity varies as a function of shear-rate, the first problem in developing the drag curve is the definition of Reynolds number since it is related to both liquid viscosity and bubble velocity. The ideal definition of the Reynolds number should satisfy the following requirements [9]: (1) the shear-thinning effect is reflected in the Reynolds number, and (2) the Reynolds number for a shear-thinning fluid reduces to that for a Newtonian fluid if the shear-thinning effect vanishes. $R e_{\mathrm{M}}$ is thus defined by introducing $2 U_{\mathrm{T}} / d_{\mathrm{e}}$ (where $U_{\mathrm{T}}$ and $d_{\mathrm{e}}$ are the terminal velocity and the volume-equivalent diameter of a bubble or drop, respectively) as a representative shear-rate in the system:

$R e_{\mathrm{M}}=\frac{\rho_{\mathrm{l}} d_{\mathrm{e}} U_{\mathrm{T}}}{\eta_{0} /\left[1+\left(\lambda\left(2 U_{\mathrm{T}} / d_{\mathrm{e}}\right)\right)^{2}\right]^{(1-n) / 2}}=\frac{\rho_{\mathrm{l}} d_{\mathrm{e}} U_{\mathrm{T}}}{C \eta_{0}}$

with

$C=\frac{1}{\left[1+\left(\lambda\left(2 U_{\mathrm{T}} / d_{\mathrm{e}}\right)\right)^{2}\right]^{(1-n) / 2}}$

where $C \eta_{0}$ means the effective viscosity of the system. If $C=1.0$, the bubble motion can be regarded as that in a Newtonian liquid.

\subsection{Level set approach for fluid flow}

A smooth scalar function denoted as $\phi$ is introduced into the formulation of a multiphase flow system to define and capture the interface between two fluids, which is identified as the zero level set of the level set function $\phi$ defined on the entire computational domain. The function $\phi$ is chosen as the signed algebraic distance to the interface, being positive in the continuous fluid phase and negative in the bubble. The following Hamilton-Jacobi type evolution equation [14] can be used to advance the level set function exactly as the bubble moves:

$\frac{\partial \phi}{\partial t}+\nabla \cdot(\boldsymbol{u} \phi)=\frac{\partial \phi}{\partial t}+(\boldsymbol{u} \cdot \nabla) \phi=0$

After introducing the level set formulation, the motion in two separate domains for two immiscible fluids may easily be formulated as a single one.

The curvature of free surface $\kappa(\phi)$ is expressed as

$\kappa(\phi)=\nabla \cdot \boldsymbol{n}=\nabla \cdot\left(\frac{\nabla \phi}{|\nabla \phi|}\right)$

where $\mathbf{n}$ is the unit vector normal to the interface pointing towards the continuous phase, and $\delta_{\varepsilon}(\phi)$ the regularized delta function defined used in Eq. (2) is

$\delta_{\varepsilon}(\phi)= \begin{cases}\frac{1}{2 \varepsilon}(1+\cos (\pi \phi / \varepsilon)) & \text { if }|\phi|<\varepsilon \\ 0 & \text { otherwise }\end{cases}$ where $\varepsilon$ prescribes the finite "half thickness" of the interface. We take $\varepsilon=1.5 \Delta z$, where $\Delta z$ is the dimensionless uniform mesh size near the interface. $H_{\varepsilon}(\phi)$ is the regularized Heaviside function expressed as

$H_{\varepsilon}(\phi)= \begin{cases}0 & \text { if } \phi<-\varepsilon \\ (1 / 2)(1+(\phi / \varepsilon)+\sin (\pi \phi / \varepsilon) / \pi) & \text { if }|\phi| \leq \varepsilon \\ 1 & \text { if } \phi>\varepsilon\end{cases}$

for avoiding the sharp change in pressure and diffusion term at the interface due to large density and/or viscosity ratios. Thus, the density and viscosity of the two phases are written as

$\rho(\phi)=\rho_{\mathrm{g}}+\left(\rho_{\mathrm{l}}-\rho_{\mathrm{g}}\right) H_{\varepsilon}(\phi)$

$\eta(\phi)=\eta_{\mathrm{g}}+\left(\eta_{\mathrm{l}}(\dot{\gamma})-\eta_{\mathrm{g}}\right) H_{\varepsilon}(\phi)$

The level set function $\phi$ is advanced with the local liquid velocity at each time step. Maintaining $\phi$ as a distance function is essential for providing the interface with an invariant bubble volume, but $\phi$ becomes gradually deviated from the distance function (i.e., $|\nabla \phi| \neq 1$ ) after some iterations. This problem can be resolved by adopting a reinitialization method proposed by Sussman et al. [15] to keep the solution accurate and maintain mass conservation of the bubble by solving the following problem to steady state in a virtual time domain:

$\frac{\partial \phi}{\partial \xi}=\operatorname{sgn}\left(\phi_{0}\right)(1-|\nabla \phi|)$

$\phi(z, 0)=\phi_{0}(z)$

where $\xi$ is the virtual time for reinitialization, $\phi_{0}(z)$ is the level set function at any computational instant, and $\operatorname{sgn}\left(\phi_{0}\right)$ is the sign function for enforcing $\nabla \phi=1$. Eq. (16) has the property of making $\phi$ remain unchanged at the interface, so the zero level set of $\phi_{0}$ and $\phi$ is the same. Away from the interface $\phi$ will converge to $|\nabla \phi|=1$, i.e., the actual distance function. In this paper, the area-preserving reinitialization procedure by Yang and Mao [16] for $\phi$ is coupled with Eq. (16) to guarantee the mass conservation by solving a perturbed Hamilton-Jacobi equation proposed by Zhang et al. [17] to pseudo-steady state in each time step. The improved reinitialization procedure can maintain the level set function as a distance function and guarantee the bubble mass conservation.

\subsection{Boundary conditions}

The boundary conditions at the axis (AD) and wall (BC) are as follows (refer to Fig. 1):

$\frac{\partial u}{\partial r}=v=0 \quad$ at $r=0$

$u=v=0 \quad$ at $r=R$

The bottom and top of the computational domains (DC and $A B$ ) are subject to the following conditions:

$\frac{\partial u}{\partial z}=\frac{\partial v}{\partial z}=0 \quad$ at $z=0$

$\frac{\partial u}{\partial z}=\frac{\partial v}{\partial z}=0 \quad$ at $z=L$

\subsection{Computational system}

The control volume formulation with the SIMPLEC algorithm [18] incorporated is used to solve the governing equations and the level set evolution equation. The power-law scheme is adopted for the discretization of the governing equations in a staggered non-uniform grid with more cells allocated densely near the interface. In order to ensure variables more accurately interpolated and 


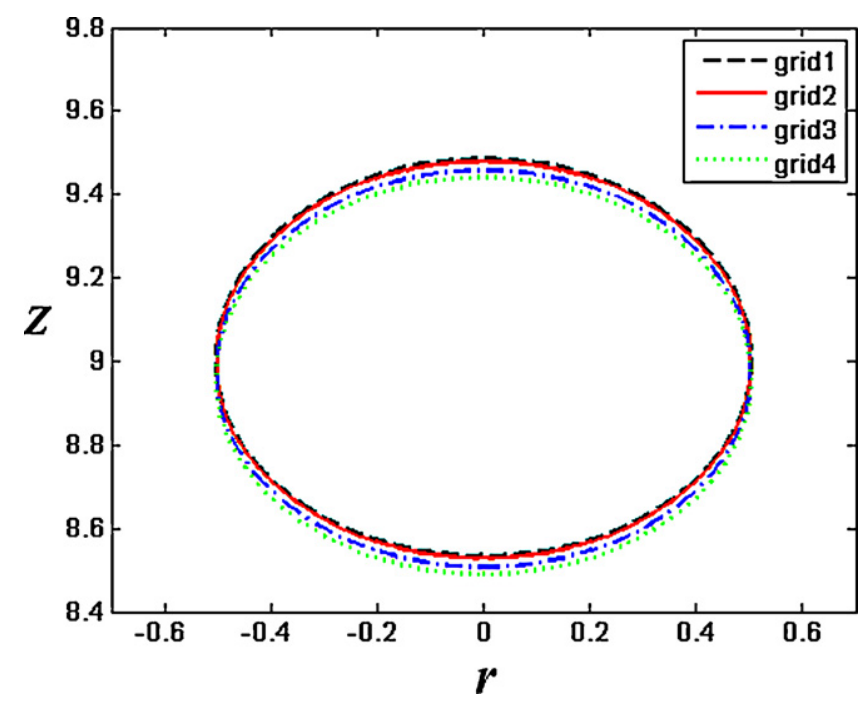

Fig. 2. Effects of mesh size on the simulated bubble shape $\left(d_{\mathrm{e}}=3.832 \mathrm{~mm}, n=0.9\right.$, $\lambda=0.207 \mathrm{~s}$ and $\eta_{0}=0.122 \mathrm{~Pa} \mathrm{~s}, R e_{\mathrm{M}}=6.65$ ).

resolved, a double fine grid is also applied. The detailed numerical method and technique were described by Sussman et al. [15] and Yang and Mao [16]. In the initial set-up, a spherical bubble with radius $R$ is set in the middle of the $z$-axis and the computational domain with $H=40 R$ and $L=15 R$ is wide enough to avoid the wall effect. Both the liquid and the bubble are assumed to be stationary at the initial state.

In this simulation, the mesh sensitivity test was performed by simulating the bubble rising under the same conditions $\left(d_{\mathrm{e}}=3.832 \mathrm{~mm}, n=0.9, \lambda=0.207 \mathrm{~s}, \eta_{0}=0.122 \mathrm{Pas}\right.$ and $R e_{\mathrm{M}}=6.65$ ) using different sized background meshes to ensure the numerical results independence of computational meshes. The effects of mesh size on predicted terminal bubble shapes are shown in Fig. 2 . Nonuniform grids $(H \times L)$ of $224 \times 97$ (grid 4), $252 \times 107$ (grid 3), $340 \times 127$ (grid 2), $380 \times 147$ (grid 1) (the last is referred to as the fine one) were tested for the simulation. A grid with $340 \times 127$ nodes is considered sufficient for spatial computational accuracy, and adopted for the subsequent simulations.

\section{Experimental}

The experiments in this work were conducted under atmospheric pressure. A schematic of the experimental apparatus is shown in Fig. 3. To help visualizing the motion of gas bubbles, a
Lucite column was constructed. It is square cross-sectioned (side length $0.21 \mathrm{~m}$ ) and $0.6 \mathrm{~m}$ high, and the column is thought large enough so as to minimize the wall effect [1].

The general procedure for a series of experimental runs is as follows: the viscous liquid that had been sufficiently exposed to room temperature was put into the column. With the help of a precision syringe pump (Harvard microprocessor multiple syringe pump, USA) enabling accurate control of gas flow, air at room temperature was introduced at a low rate and single bubbles were formed at a stainless steel nozzle with a flat opening. The nozzle was located at the center, $3 \mathrm{~cm}$ above the bottom. Various nozzles with different inner diameters were used to generate single bubbles with desired sizes. Time interval between subsequent bubbles was sufficiently long (>100 s) to minimize the mutual influence between successive bubbles. Since the single bubbles in the present experiments were roughly spherical and axisymmetric, rising rectilinearly, the images of rising bubbles were recorded using a single high-speed CCD camera (LG CCD GC-145C-G, Korea) with the shutter speed variable between $1 / 60$ to $1 / 100,000$ s. The instantaneous positions of a bubble was read out from each frame of images with the resolution of $752 \times 582$ pixels against a precision scale along the column wall. The local velocity was calculated by a frame-to-frame analysis of successive images using imaging software (Photoshop 8.0). We assumed that the velocity was terminal when it remained constant (within 5\%) in at least five consecutive frames.

Sodium aqueous carboxymethyl cellulose (CMC) sodium salt, sodium hydroxyl-ethyl cellulose (HEC) and xanthan gum (XG) solutions were used as the ambient shear-thinning liquids (continuous phase). Solutions were maintained at $21^{\circ} \mathrm{C}$ during the experiments. The liquid densities were measured using a balance hydrometer and none were found to be significantly different from that of water because the concentration of these polysaccharides is very low. The surface tension of each mixture was measured with a tensiometer by the du Nouy Ring method. The surface tension of the solution did not change significantly as a function of polysaccharides concentration within the concentration ranges used in this study and remained close to that of water $\left(7.2 \times 10^{-2} \mathrm{~N} \mathrm{~m}^{-1}\right)$.

\section{Results and discussion}

\subsection{Bubble shape and rising velocity in Newtonian fluids}

In the past decades, many experimental studies were focused on the motion of bubbles and drops. The main practical interest is the determination of the fluid particle shape and terminal velocity at steady state motion. The predicted bubble shapes and terminal steady state conditions are compared with the experimen-

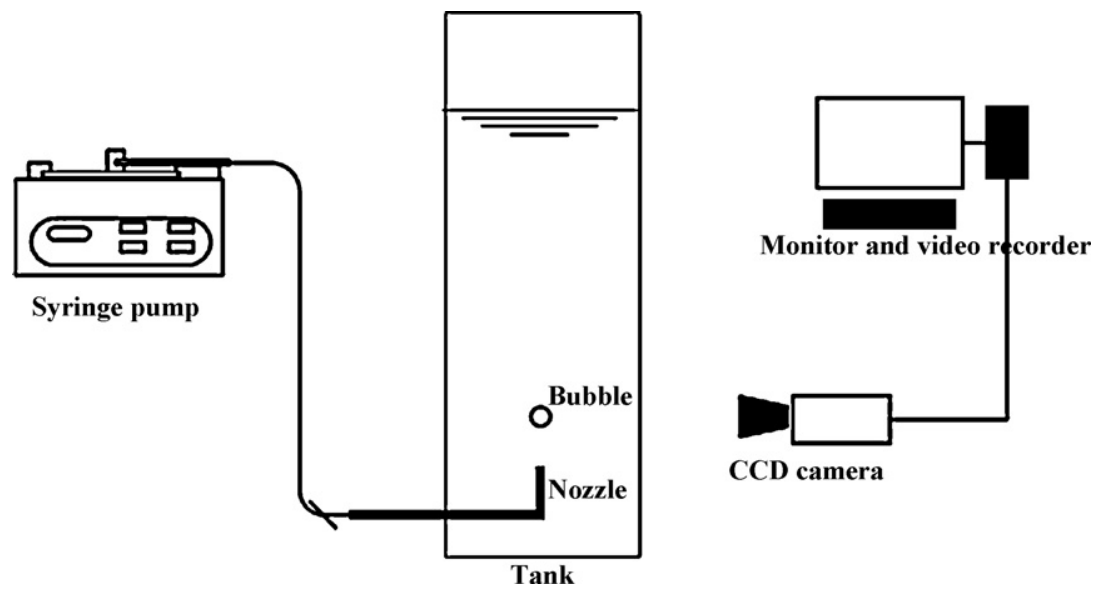

Fig. 3. Schematic diagram of experimental setup. 
Table 1

Comparison of bubble shapes and terminal Reynolds numbers observed in experiments [19] and the present predictions in Newtonian fluids.

\begin{tabular}{|c|c|c|c|c|}
\hline \multirow[t]{2}{*}{ Test case } & \multicolumn{2}{|l|}{ Experiment [19] } & \multicolumn{2}{|l|}{ Simulation } \\
\hline & $\begin{array}{l}\text { Test } \\
\text { conditions }\end{array}$ & $\begin{array}{l}\text { Observed bubble } \\
\text { terminal shapes }\end{array}$ & $\begin{array}{l}\text { Predicted bubble } \\
\text { terminal shapes }\end{array}$ & $\begin{array}{l}\text { Predicted Reynolds } \\
\text { numbers }\end{array}$ \\
\hline 1 & $M o=848 R e=2.47$ & & & $R e=2.35$ \\
\hline 2 & $M o=266 R e=3.57$ & & & $\operatorname{Re}=3.7$ \\
\hline 3 & $M o=41.1 R e=7.16$ & & & $R e=7.05$ \\
\hline 4 & $M o=5.51 R e=13.3$ & & & $R e=13.45$ \\
\hline 5 & $M o=1.31 R e=20.4$ & & & $R e=20.72$ \\
\hline
\end{tabular}

Note: $E o=116$.

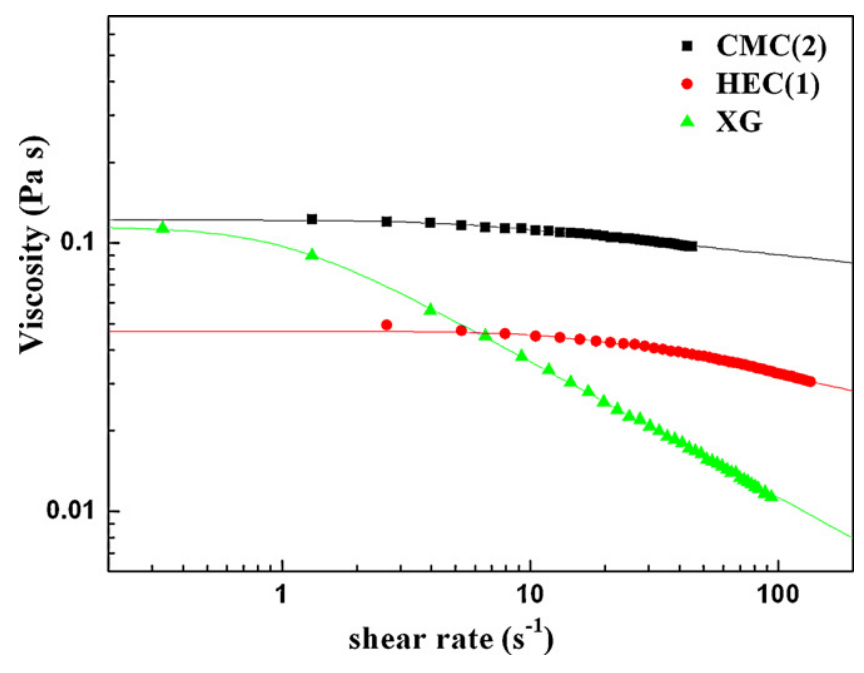

Fig. 4. Viscosities as a function of shear rates for three test fluids $\left(T=21^{\circ} \mathrm{C}\right)$.

tal results reported by Bhaga and Weber [19]. Their experimental results were presented in the terms of Eotvos number $(E o=$ $\left.d_{\mathrm{e}}^{2} \rho_{\mathrm{l}} g / \sigma\right)$, Morton number $\left(M o=g \mu_{1}^{4} /\left(\rho_{1} \sigma^{3}\right)\right)$ and Reynolds number $\left(R e=\rho_{\mathrm{l}} U d_{\mathrm{e}} / \mu\right)$. The comparison of steady-state cases in Table 1

Table 2

Parameters for Carreau model and physical properties of liquids.

\begin{tabular}{llllll}
\hline Liquid & $\eta_{0}($ Pa s $)$ & $\lambda(\mathrm{s})$ & $n$ & $\rho_{1}\left(\mathrm{~kg} \mathrm{~m}^{-3}\right)$ & $\sigma\left(\mathrm{mN} \mathrm{m}^{-1}\right)$ \\
\hline CMC1 & 0.0377 & 0.512 & 0.89 & 1000.0 & 72 \\
CMC2 & 0.122 & 0.207 & 0.90 & 1000.0 & 72 \\
HEC1 & 0.047 & 0.0624 & 0.79 & 1000.0 & 72 \\
HEC2 & 0.315 & 0.260 & 0.68 & 1000.0 & 72 \\
XG & 0.115 & 0.984 & 0.49 & 1000.0 & 72 \\
\hline
\end{tabular}

Table 3

Comparisons between experimental and numerical results for a single bubble rising in Carreau shear-thinning liquids.

\begin{tabular}{llccrcl}
\hline Liquid & $d_{\mathrm{e}}(\mathrm{mm})$ & $U_{\mathrm{T}, \exp }\left(\mathrm{cm} \mathrm{s}^{-1}\right)$ & $U_{\mathrm{T}, \mathrm{cal}}\left(\mathrm{cm} \mathrm{s}^{-1}\right)$ & $R e_{\mathrm{M}, \exp }$ & $R e_{\mathrm{M}, \mathrm{cal}}$ & $D_{\mathrm{ev}}{ }^{*}(\%)$ \\
\hline CMC1 & 4.19 & 21.5 & 20.87 & 121.27 & 116.29 & 2.9 \\
$\mathrm{CMC2}$ & 3.96 & 9.88 & 9.39 & 4.05 & 3.832 & 4.4 \\
HEC1 & 2.84 & 11.88 & 11.6 & 10.19 & 9.91 & 2.4 \\
HEC2 & 3.11 & 5.25 & 4.74 & 1.041 & 0.91 & 9.7 \\
XG & 2.29 & 26.5 & 24.6 & 84.07 & 75.15 & 7.16 \\
\hline
\end{tabular}

${ }^{*} D_{\mathrm{ev}}=\left|U_{\mathrm{cal}}-U_{\mathrm{exp}}\right| / U_{\mathrm{exp}} \times 100$.

indicates good agreement between computational and experimental results. These simulations suggest that our method is effective for investigating the motion of a bubble with very steep gradients in density and viscosity across interface under the laminar flow conditions using an Eulerian grid. The method provides the basis for further investigation of non-Newtonian fluids. The physical properties of air are $\rho_{\mathrm{g}}=1.2 \mathrm{~kg} \mathrm{~m}^{-3}$ and $\eta_{\mathrm{g}}=1.8 \times 10^{-5}$ Pa s.

\subsection{Bubble rising in shear-thinning non-Newtonian fluids}

Fig. 4 shows the representative apparent viscosity vs. shear-rate data for some test fluids measured using a Brookfield Viscometer (DV-III) at ambient experimental temperature. The Carreau model

Table 4

Carreau model parameters and numerical results of bubbles with $d_{\mathrm{e}}=1 \mathrm{~cm}$.

\begin{tabular}{lllllll}
\hline System & $\eta_{0}($ Pa s $)$ & $\lambda(\mathrm{s})$ & $n$ & $U_{\mathrm{T}}\left(\mathrm{cm} \mathrm{s}^{-1}\right)$ & $2 \lambda U_{\mathrm{T}} / d_{\mathrm{e}}$ & $R e_{\mathrm{M}}$ \\
\hline 1 & 0.5 & - & 1.0 & 11.2 & - & 2.24 \\
2 & 0.5 & 1.00 & 0.8 & 16.5 & 33.0 & 6.65 \\
3 & 0.5 & 1.00 & 0.5 & 22.7 & 45.4 & 30.69 \\
4 & 0.5 & 1.00 & 0.4 & 23.9 & 47.8 & 48.8 \\
5 & 0.1 & 1.00 & 0.8 & 25.3 & 50.6 & 55.5 \\
6 & 0.5 & 1.00 & 0.3 & 25.6 & 51.2 & 80.85 \\
\hline
\end{tabular}


Eq. (7) is fitted to the experimental data, which is represented in Fig. 4 by full lines. The other solutions studied are not plotted for better visualization. All the solutions exhibited a shear-thinning behaviour. The parameter values of the Carreau model and other physical properties under test conditions are listed in Table 2.

In this study, the terminal rising velocity of air bubbles in 5 nonNewtonian liquids with shear-thinning property are determined and these bubbles are simulated using the present numerical program. Table 3 shows the numerical and experimental results for a single bubble rising freely in pure shear-thinning non-Newtonian solutions. The calculated terminal velocities are in close agreement with the present experimental values with the relative deviation below $10 \%$. The satisfactory agreement indicates the validity of numerical procedure for the numerical simulation of a single bub- (a)
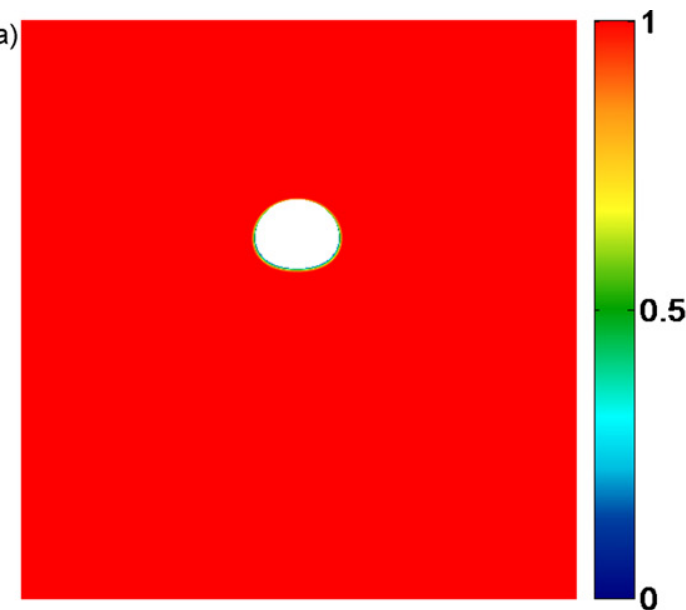

(c)

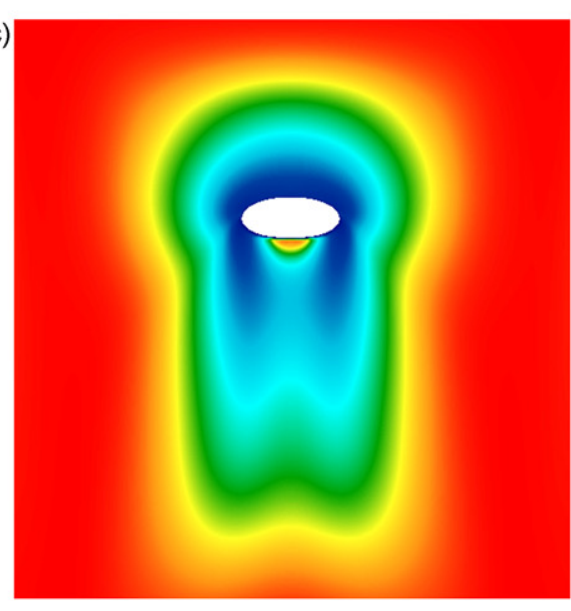

(e)
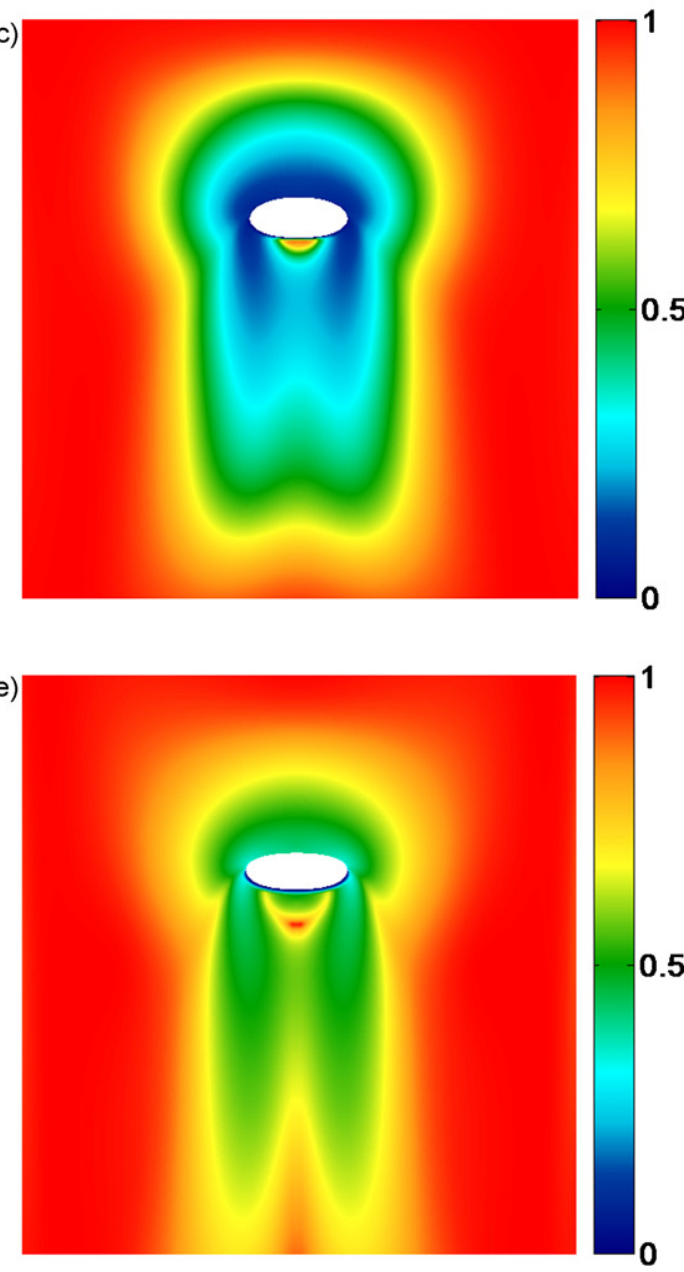

(b)

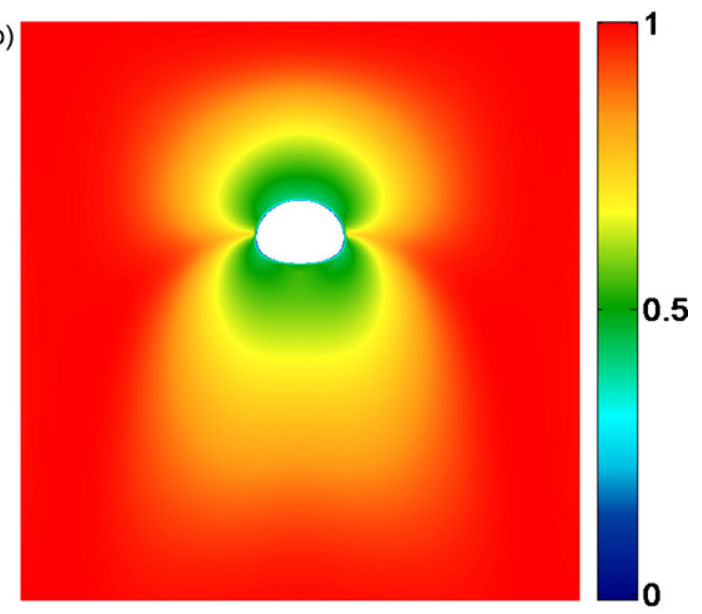

(d)

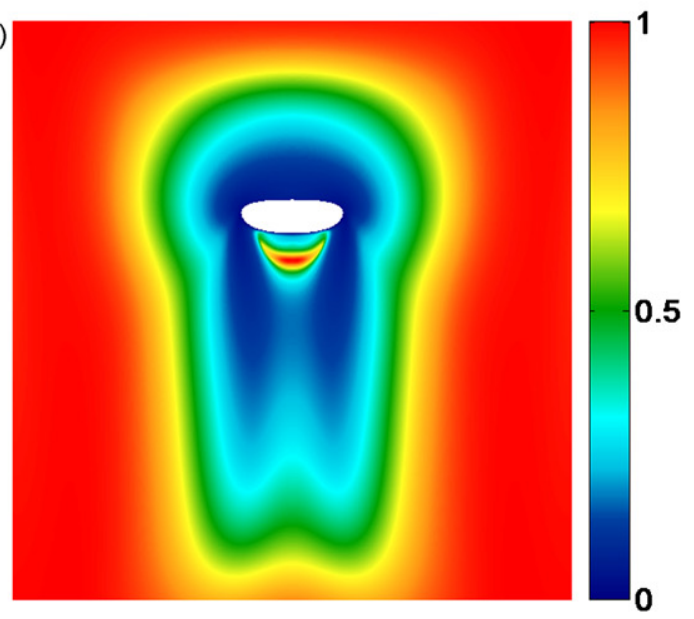

(f)

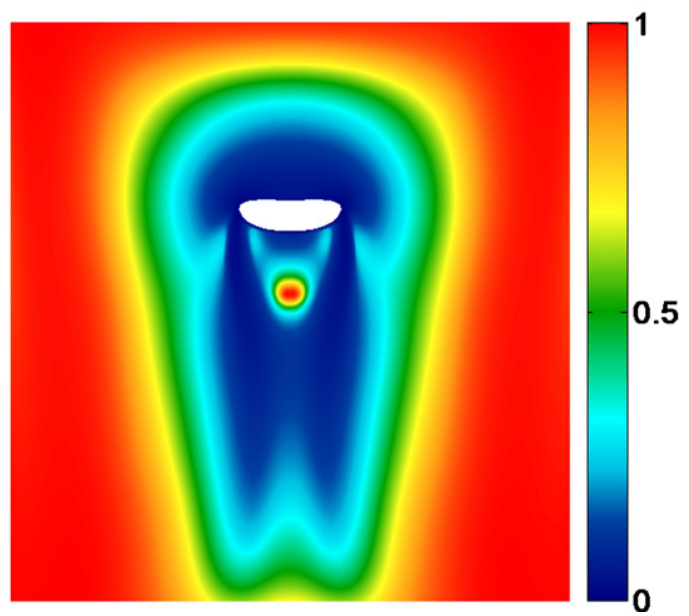

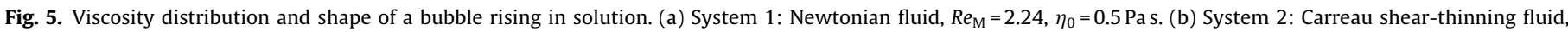

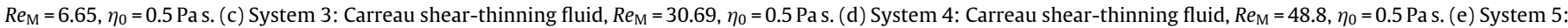
Carreau shear-thinning fluid, $R e_{\mathrm{M}}=55.5, \eta_{0}=0.1$ Pa s. (f) System 6: Carreau shear-thinning fluid, $R e_{\mathrm{M}}=80.85, \eta_{0}=0.5 \mathrm{~Pa}$. 
ble rise in a shear-thinning non-Newtonian continuous phase. In these shear-thinning solutions the apparent viscosity around the bubble decreases due to the shear, so the bubble can rise more and more easily. It is interesting to note that the zero-shear viscosity of CMC2 and XG is roughly equal (Table 2 ), but $U_{\mathrm{T}}$ of the bubble in
XG is much larger than that in $\mathrm{CMC2}$, since XG has stronger shearthinning property and the liquid around the bubble thus becomes less viscous. Hence, understanding the viscosity distribution over the whole domain is important to elucidate the bubble behaviors in shear-thinning non-Newtonian liquids. (a)
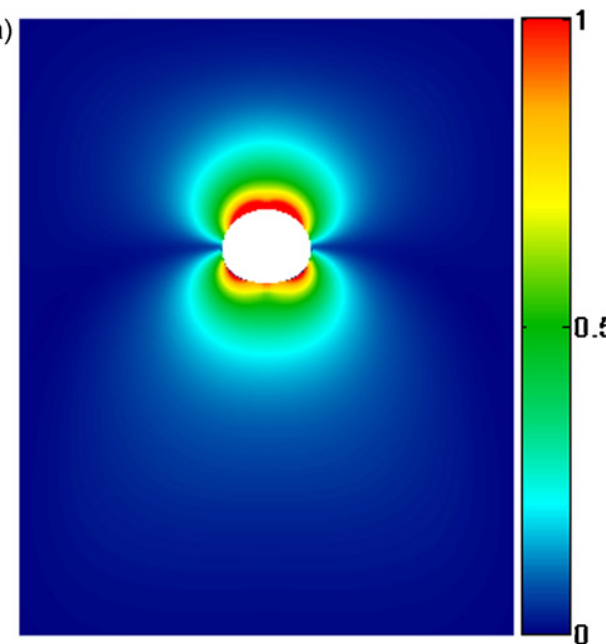

(c)

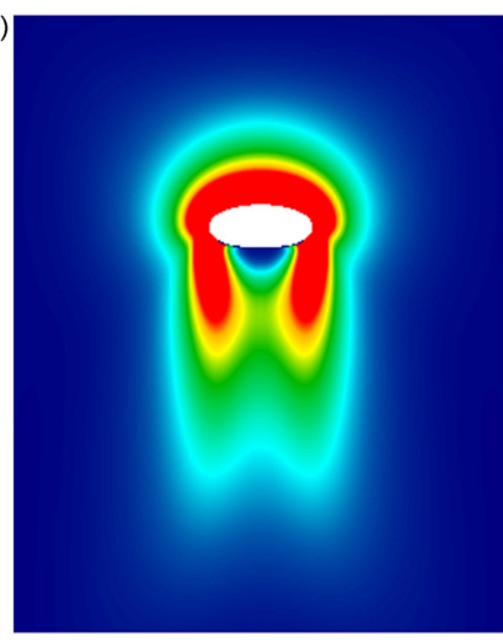

(e)

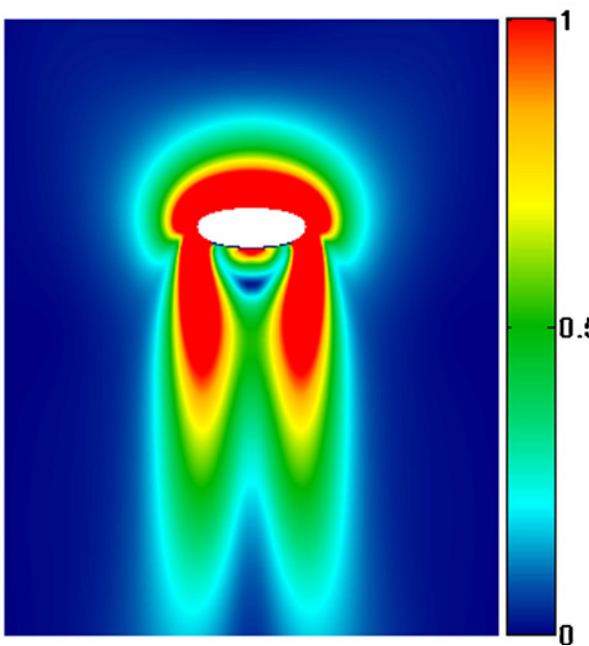

(b)

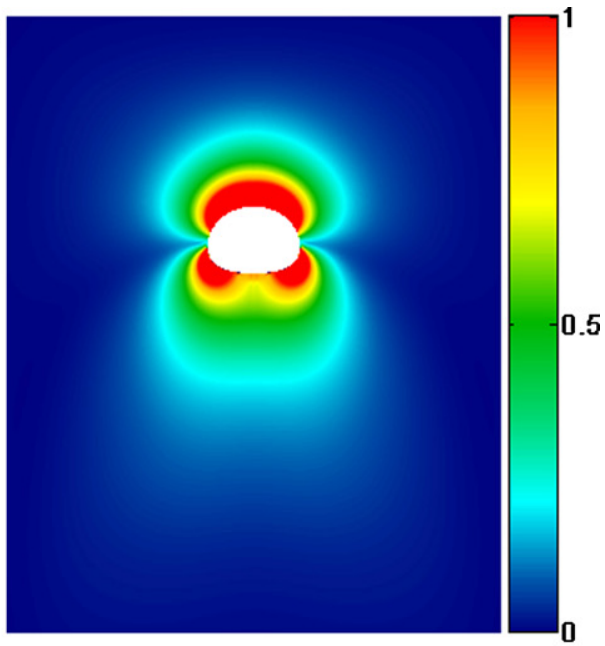

(d)

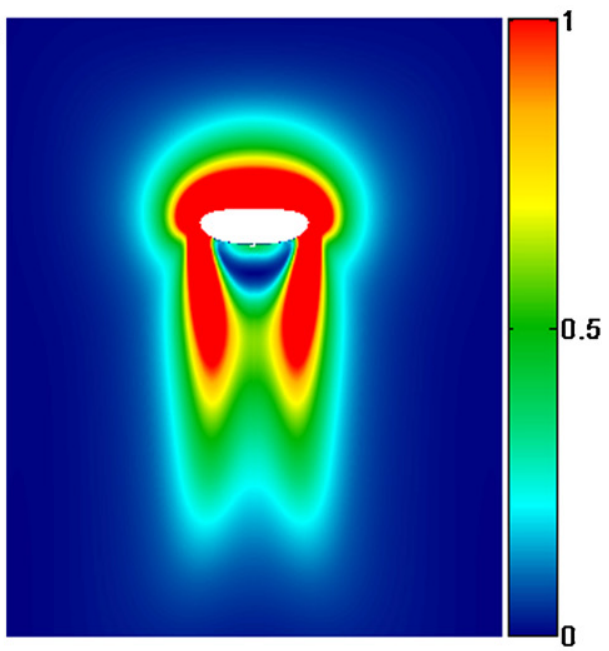

(f)

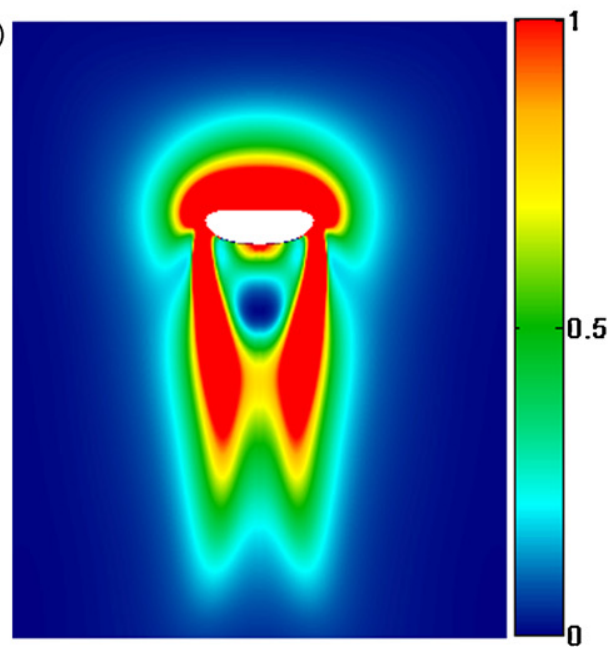

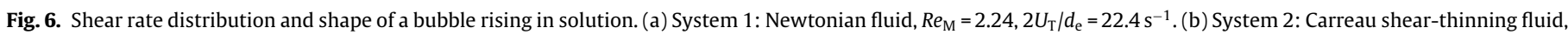

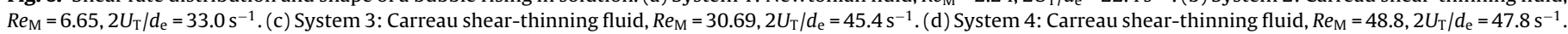
(e) System 5: Carreau shear-thinning fluid, $R e_{\mathrm{M}}=55.5,2 U_{\mathrm{T}} / d_{\mathrm{e}}=50.6 \mathrm{~s}^{-1}$. (f) System6: Carreau shear-thinning fluid, $R e_{\mathrm{M}}=80.85,2 U_{\mathrm{T}} / d_{\mathrm{e}}=51.2 \mathrm{~s}^{-1}$. 


\subsection{Viscosity distribution around a bubble}

It is difficult to explore the distribution of viscosity by experimental techniques. However, the local viscosity change around the bubble can be derived in detail from the numerical results. In this study, the shear-thinning liquids whose properties are shown in Table 4 are tested in order to elucidate the shear-thinning effect on bubble rising. The numerical results are also listed in Table 4 . The gas-related physical properties of the systems are $\rho_{\mathrm{g}}=1.2 \mathrm{~kg} \mathrm{~m}^{-3}$, $\eta_{\mathrm{g}}=1.8 \times 10^{-5}$ Pa and $\sigma=7.2 \times 10^{-2} \mathrm{~N} \mathrm{~m}^{-1}$.

Fig. 5 shows the viscosity distribution (non-dimensionalized by $\eta_{0}$ ) around a bubble for systems 1 through 6 . The colorbar shown at the right of the figures depicts the range of reduced apparent viscosity around the bubble. The real shape of the bubbles is also displayed in Fig. 5. System 1 corresponds to the bubble rising in Newtonian fluid with the same zero shear-rate viscosity and the viscosity around the bubble is constant. As for systems 2 through 6, the viscosity around the bubble varies in correspondence to the bubble shape and the shear-thinning property of the liquid, and the degree of the decrease in viscosity is the largest in close vicinity to the bubble. The change in viscosity becomes gradually drastic from system 3 to system 6 , showing that a confined region with high viscosity exists in the wake of these bubbles and it becomes finally detached from the bubble rear surface. As seen in Table 4, it is obvious that $U_{\mathrm{T}}$ (or $R e_{\mathrm{M}}$ ) increases gradually due to the stronger shear-thinning effect. The bubble can rise faster due to the large extent of decrease in viscosity around the bubble. Obviously, the bubble shape in the shear-thinning non-Newtonian fluid differs much from that in the Newtonian case. As the shear-thinning effect becomes intensive gradually, the bubble takes the more oblate shape, with the front surface flatter than the rear part.

As the apparent viscosity of the shear-thinning fluids decreases due to the increased shear-rate $\dot{\gamma}$,

$\dot{\gamma}=\left[2\left(\frac{\partial u}{\partial z}\right)^{2}+2\left(\frac{\partial v}{\partial r}\right)^{2}+\left(\frac{\partial v}{\partial z}+\frac{\partial u}{\partial r}\right)^{2}+2\left(\frac{v}{r}\right)^{2}\right]^{1 / 2}$

its distribution, which can be calculated from the predicted velocity field $\mathbf{u}$, sheds light for understanding the local apparent viscosity. As shown in Fig. 6, the shear rate distribution around a bubble in the Newtonian (Fig. 6a) and non-Newtonian (Fig. 6b) liquids is similar when the shear-thinning effect is weak and the Reynolds number is small. However, a larger area of higher local shear rate at the bubble nose in Fig. 6b can be observed in the shear-thinning liquid than that in Fig. 6a, as a joint effect of shear-thinning property and a larger $R e_{\mathrm{M}}$ in Fig. 6b. As demonstrated in Fig. 6c-f, a region of much lower shear-rate is at the rear of the bubble, which corresponds to a much higher viscosity region in the wake of the bubble. In these strong shear-thinning cases, the topology
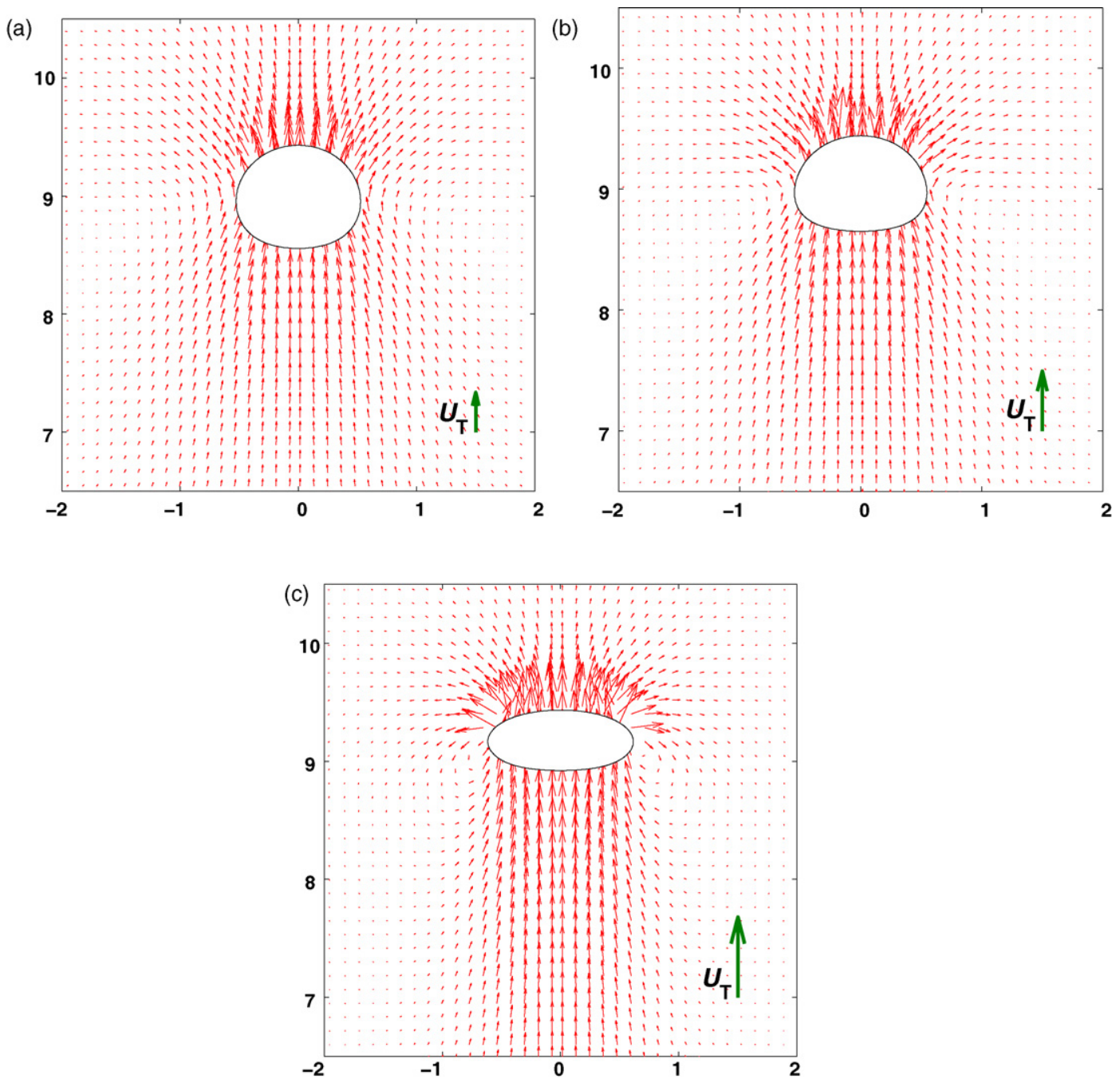

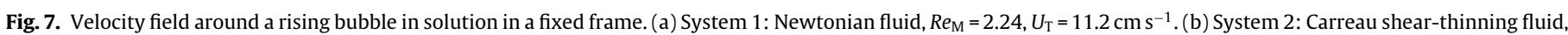
$R e_{\mathrm{M}}=6.65, U_{\mathrm{T}}=16.5 \mathrm{~cm} \mathrm{~s}^{-1}$. (c) System 3: Carreau shear-thinning fluid, $R e_{\mathrm{M}}=30.69, U_{\mathrm{T}}=22.7 \mathrm{~cm} \mathrm{~s}^{-1}$. 
of shear rate distribution resembles its counterpart viscosity distribution in Fig. 5. As the shear-thinning effect becoming stronger in Fig. 6e and f, the low shear region even becomes isolated amid the wake, detached from the bubble rear. It indicates clearly that there exists a higher shear-rate region in front of the bubble and a streaming toroidal one at the outer region in the wake, where the viscosity is subject to drastic decrease correspondingly. In Fig. 6, the shear rate has been non-dimensionalized with the respective maximum value of $\dot{\gamma}$ in the flow field to show the pattern of variation.

Although the viscosity of shear-thinning fluids is not directly related to the liquid velocity field, it provides the relevant information for understanding the distribution of the apparent local viscosity. The flow field in the fixed reference frame around a bubble is shown in Fig. 7. The velocity distribution around the bubble rising in the Newtonian fluid is classical: the rising bubble pushes the liquid upwards in front of it and the liquid flows back into the bubble wake, and a toroidal vortex is formed on the side of the bubble with approximate fore-aft symmetry (Fig. 7a). The flow is quite similar in the non-Newtonian cases despite its shear-thinning feature, but the vortex appears with increased shear-thinning effect and is pushed farther behind the equatorial plane (Fig. 7b and c). The flow field shows significant velocity gradient in front of the bubble, which corresponds well to the high shear-rate region at the front. These velocity fields are similar to the flow visualization with the help of a 3D PIV (Particle Image Velocimetry) system by Funfschilling and Li [20].

It is also interesting to view and analyze the flow field in the reference coordinate system moving with the bubble. The relative velocity in the liquid domain is given by

$\boldsymbol{u}_{\mathrm{R}}(z)=\boldsymbol{u}(z)-\boldsymbol{i} U_{\text {bubble }}$

with the rectilinear bubble rising velocity calculated as the average over the whole bubble surface $\Omega$ :

$U_{\text {bubble }}=\frac{\int_{\phi=0} u \mathrm{~d} \Omega}{\int_{\phi=0} \mathrm{~d} \Omega}$

The integration is carried out along the bubble surface which corresponds to the zero set of the level set function. The procedure of numerical solution has been presented previously by Wang et al. in detail [21]. The field of relative velocity around the bubble is presented in Fig. 8 as velocity vector maps.

The flow pattern around the nose of the bubbles is similar for the 2 cases, and the magnitude of velocity gradient varies moderately. The more significant difference appears in the bubble wake. It is obvious that the wake region is enlarged as the value of $R e_{\mathrm{M}}$ increased gradually from Fig. $8 \mathrm{a}\left(R e_{\mathrm{M}}=30.69\right)$ to Fig. $8 \mathrm{~b}\left(R e_{\mathrm{M}}=48.8\right)$. However, it is difficult to discern if there is a circulating vortex in the wake or not.

It is easier to observe and analyze the flow structure by the streamline maps for the 5 non-Newtonian cases in Table 4 . The stream function is dimensionless, based on far upstream velocity normalized to unity using $U_{\mathrm{T}}$ and the coordinates nondimensionalized with $d_{\mathrm{e}}$. These streamline maps are presented in Fig. 9 together with that for the bubble with the same value of $R e$ in a Newtonian liquid, in an attempt to distinguish between the effect of shear-thinning property and that of increase of $R e_{\mathrm{M}}$. The simulated bubble in the Newtonian liquid has the same bubble diameter $d_{\mathrm{e}}$, terminal velocity $U_{\mathrm{T}}$ and liquid density $\rho_{1}$ as that in shear-thinning non-Newtonian liquid. However, the liquid viscosity is smaller than the zero shear-rate viscosity of the shear-thinning liquid, so as to make $R e=R e_{\mathrm{M}}$. There is no circulation in the wake in Fig. 9b(top) for the shear-thinning non-Newtonian liquid because of a high viscosity region attached closely in the bubble wake (Fig. 5c). It is easily observed that a circulating vortex appears in Fig. 9c(top), which is difficult to recognize in Fig. 8b. When there is a circulating vortex in the wake, a secondary vortex appears inside the bubbles, despite the fact that the bubble becomes flatter as $R e_{M}$ increases gradually. Only with its presence, the vortices in the wake and in the bubble become compatible with the main convective flow passing the bubble. For systems 4, 5 and 6, the wake length increases progressively as $R e_{\mathrm{M}}$ increases, and roughly matches the length of the low viscosity region in the wake in Fig. $5 \mathrm{~d}$ through $5 \mathrm{f}$.

Also noticed is the fact that at the same value of the Reynolds number, the aspect ratio (the maximum axial dimension/the maximum lateral dimension) of the bubble in non-Newtonian liquid is visibly smaller than that in Newtonian liquid, due to the shear thinning effect.

\subsection{Comparison with reported results}

Ohta et al. [10] investigated the Newtonian drop (silicone oil) flow in a shear-thinning fluid (sodium acrylate polymer, SAP) with the Carreau-Yasuda model Eq. (6) and the VOF (Volume-of-Fluid) method. In this work the level set method for tracking the interface is used to simulate the motion of a drop in shear-thinning nonNewtonian liquids and the predictions are compared with results of Ohta et al. [10]. The values of the Carreau-Yasuda model and
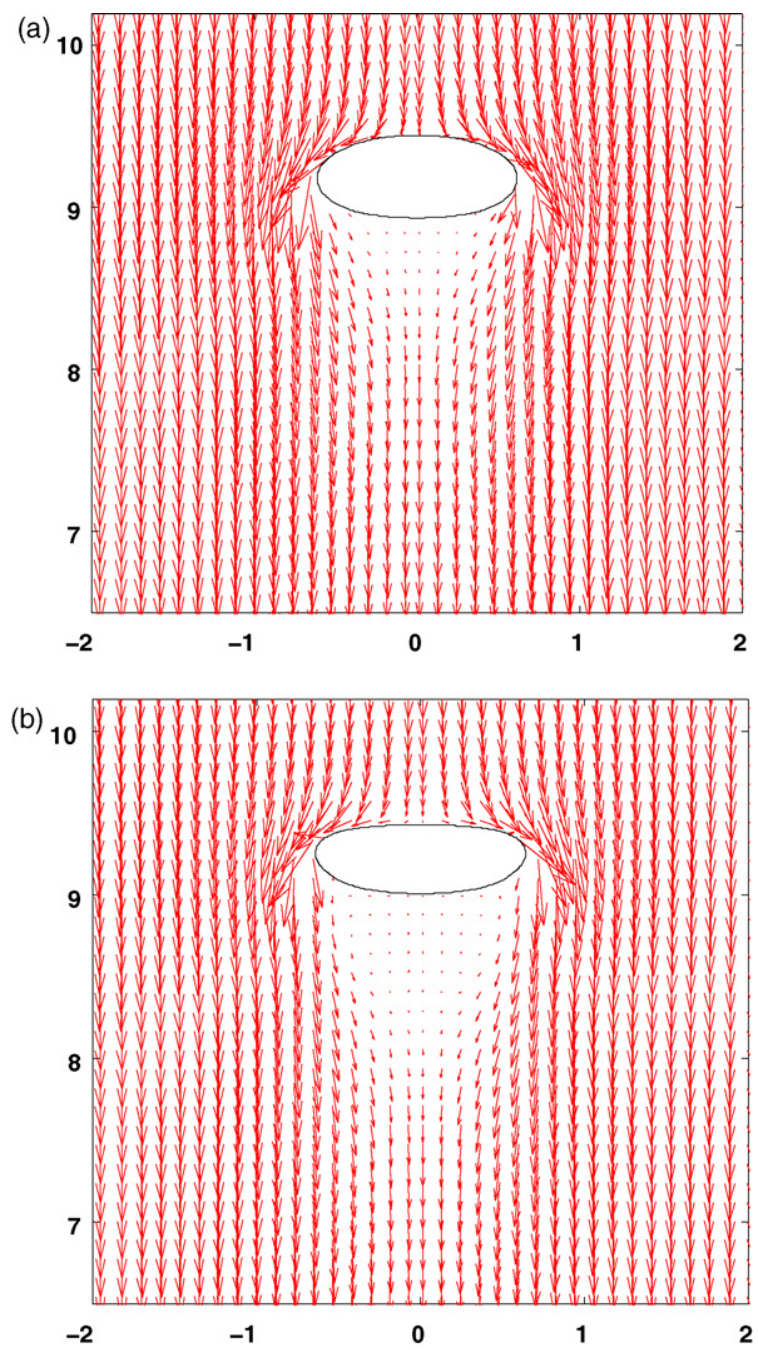

Fig. 8. Velocity field around a bubble rising in Carreau shear-thinning in a reference frame moving with the bubble. (a) System 3: Carreau shear-thinning fluid, $R e_{\mathrm{M}}=$ 30.69. (b) System 4: Carreau shear-thinning fluid, $R e_{\mathrm{M}}=48.8$. 
(a)
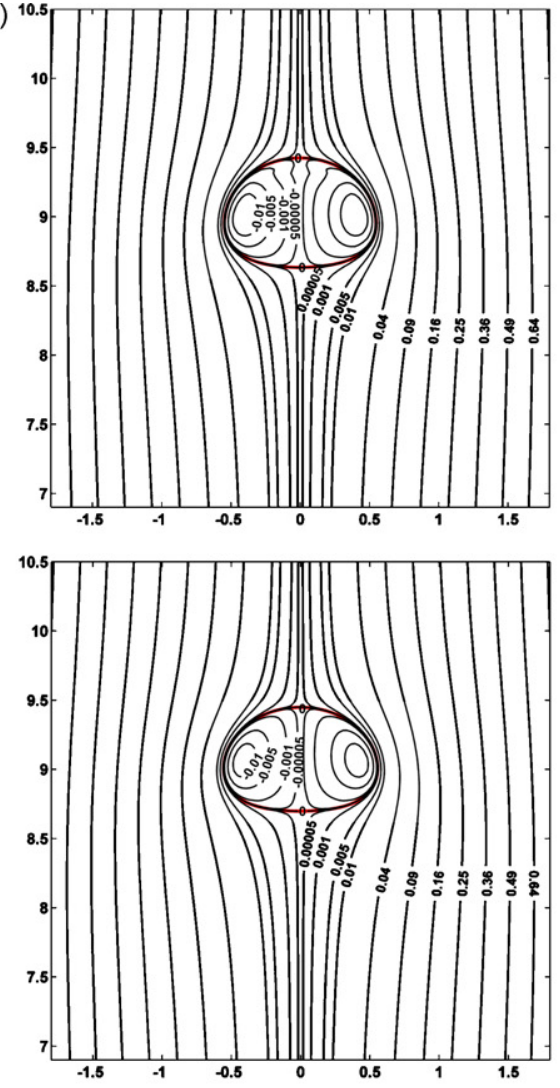

(c)
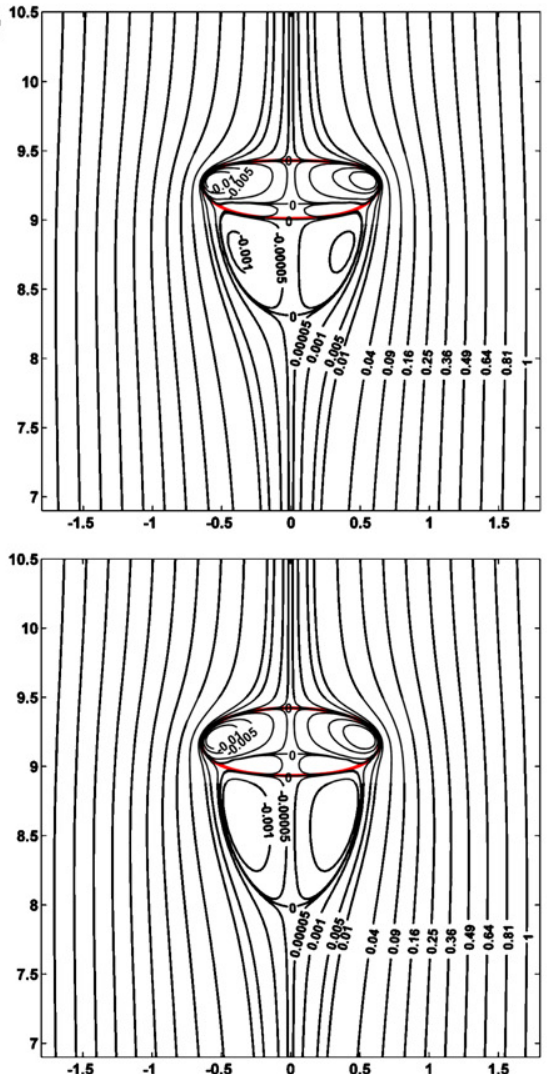

(b)
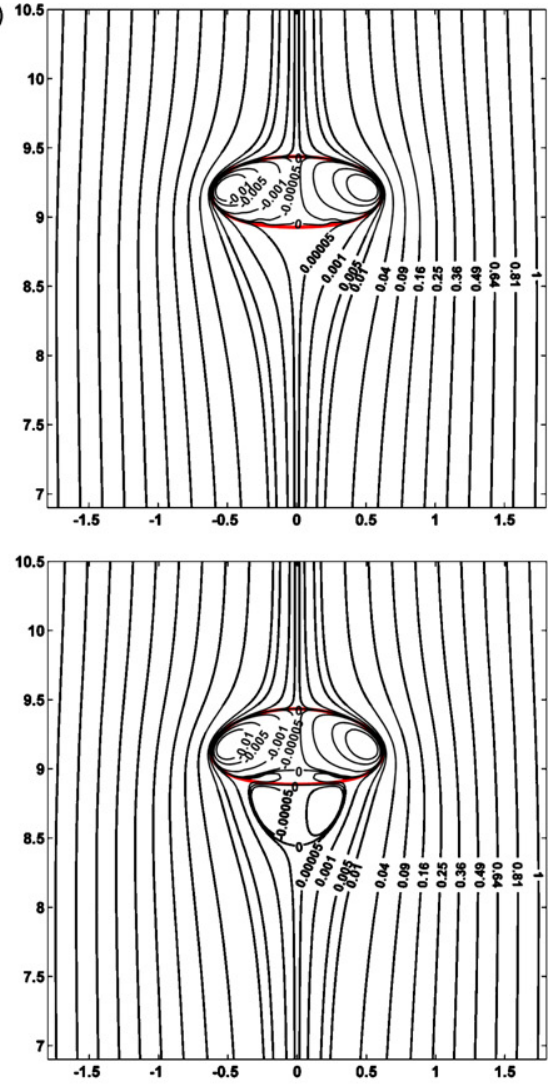

(d)
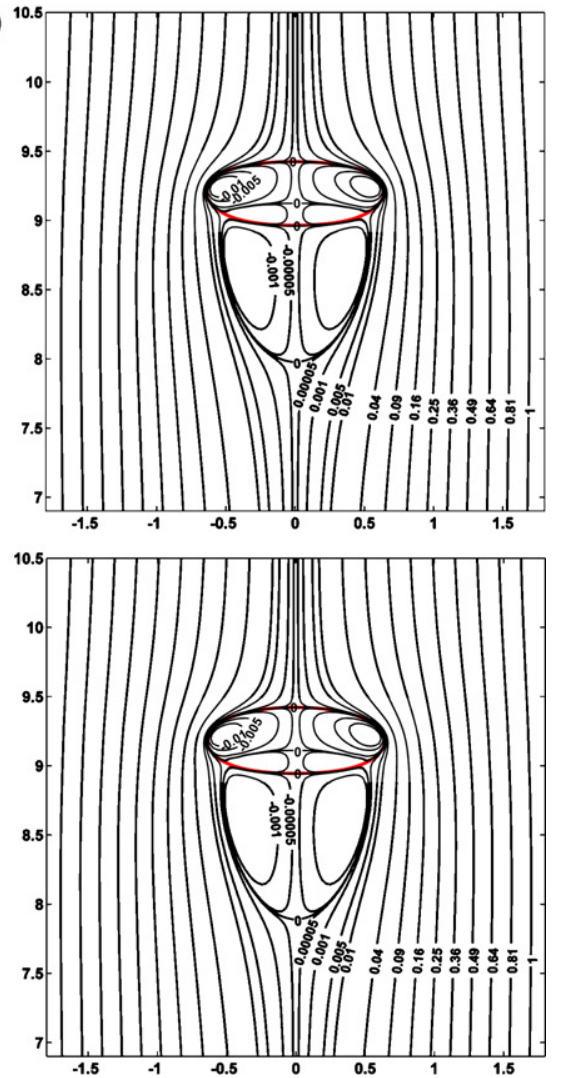

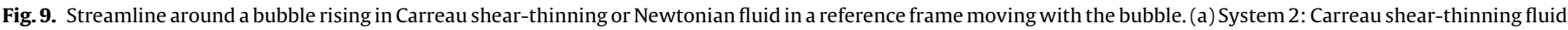

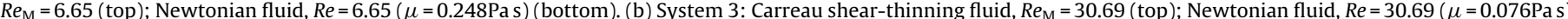

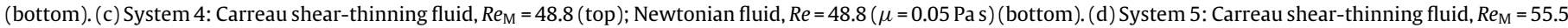

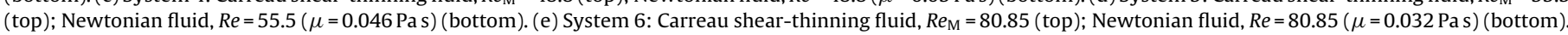


(e)
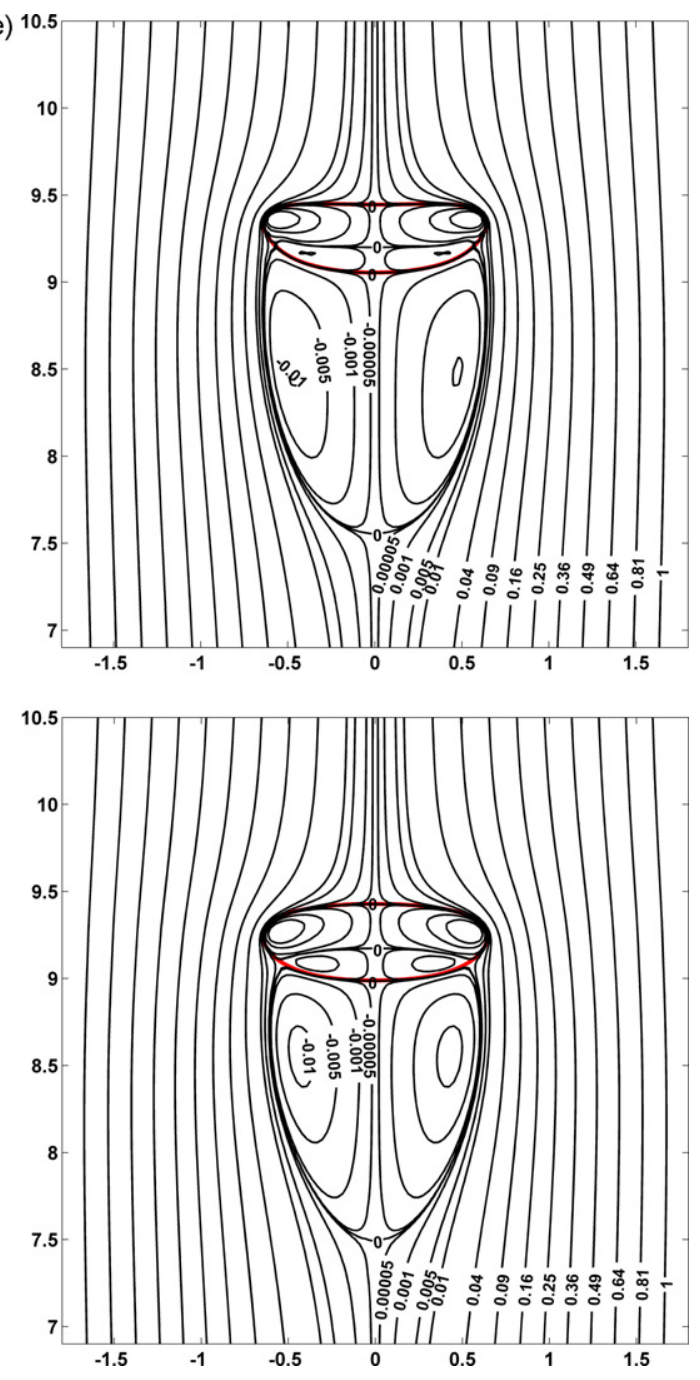

Fig. 9. (Continued).

Table 5

Carreau-Yasuda model parameters of shear-thinning liquids [10].

\begin{tabular}{lllll}
\hline Liquid & $\eta_{0}($ Pa s $)$ & $\lambda(\mathrm{s})$ & $n(-)$ & $\beta(-)$ \\
\hline SAP1 & 0.05 & 0.89 & 0.25 & 2.5 \\
SAP2 & 0.026 & 1.44 & 0.4 & 4.0 \\
\hline
\end{tabular}

other physical properties under test conditions are listed in Table 5 and Table 6 separately.

Figs. 10-12 compare the predicted viscosity distributions with those in ref. [10] for a drop rising in a SAP (sodium acrylate polymer) solution. In Figs. 10 and 11, the red area corresponds to the zero shear-rate viscosity, and the blue area denotes the low viscosity region around a drop (silicone oil with a constant value). In Fig. 12, the dark-green area corresponds to the zero shear-rate viscosity
Table 7

Comparison of the predicted results with those in ref. [10] for a drop rising in Carreau-Yasuda shear-thinning solution of SAP.

\begin{tabular}{ccccccc}
\hline Case & $d_{\mathrm{e}}(\mathrm{mm})$ & $E o$ & $U_{\mathrm{T}}\left(\mathrm{cm} \mathrm{s}^{-1}\right)$ & $R e_{\mathrm{M}}$ (level set) & $R e_{\mathrm{M}}(\mathrm{VOF})$ & $R e(\exp )$ \\
\hline 1 & 10.7 & 2.4 & 11.6 & 229 & 227 & 224 \\
2 & 5.9 & 0.6 & 5.5 & 90 & 92 & 86 \\
& 11.6 & 2.1 & 11.62 & 330 & 341 & 335 \\
3 & 9.8 & 1.0 & 6.6 & 147 & 150 & 140 \\
\hline
\end{tabular}

( 0.0260 Pa s), and the red area is the viscosity of the drop with a constant value. As a common trend in the viscosity distribution, the decreasing of viscosity at the front of a drop radiates from the drop, and the viscosity behind the drop decreases along the downstream flow. It can be verified that the computed viscosity distributions by VOF and the present level set method agree well qualitatively with each other.

Table 7 compares the results obtained from ref. [10] based on the VOF method and from this simulation using the level set method under a number of experimental test conditions. It can be found
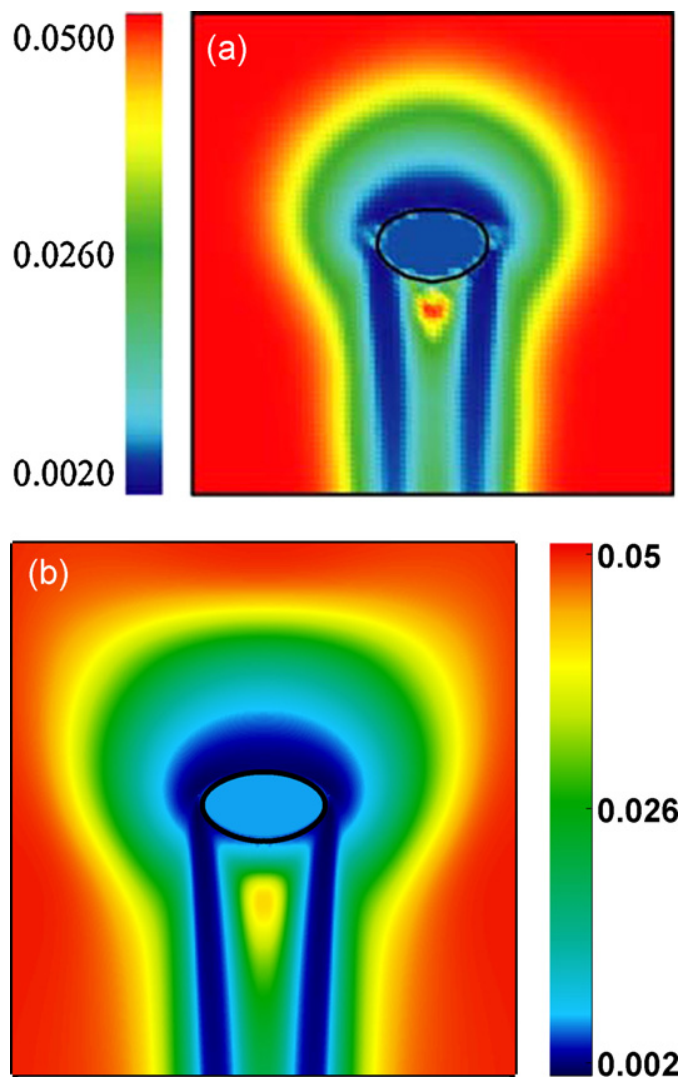

Fig. 10. Comparison of the viscosity distribution and shape of a drop rising in Carreau-Yasuda shear-thinning solution (case $1, d_{\mathrm{e}}=10.7 \mathrm{~mm}$, viscosity in unit of Pa s). (a) VOF ${ }^{[10]}$ and (b) level set method.

Table 6

Experimental cases and their physical properties [10].

\begin{tabular}{|c|c|c|c|c|c|}
\hline Case (continuous-dispersed) & $\eta_{0}(\mathrm{~Pa} \mathrm{~s})$ & $\rho_{1}\left(\mathrm{~kg} \mathrm{~m}^{-3}\right)$ & $\rho_{\mathrm{d}}\left(\mathrm{kg} \mathrm{m}^{-3}\right)$ & $\eta_{\mathrm{d}}(\mathrm{Pas})$ & $\sigma\left(\mathrm{mN} \mathrm{m}^{-1}\right)$ \\
\hline 1 (SAP1-silicone oil 5) & 0.05 & 1000.4 & 925.7 & 0.006 & 34.7 \\
\hline 2 (SAP2-silicone oil 10) & 0.026 & 1001.4 & 945.0 & 0.012 & 34.7 \\
\hline 3 (SAP2-silicone oil 50) & 0.026 & 1001.4 & 971.0 & 0.062 & 29.5 \\
\hline
\end{tabular}



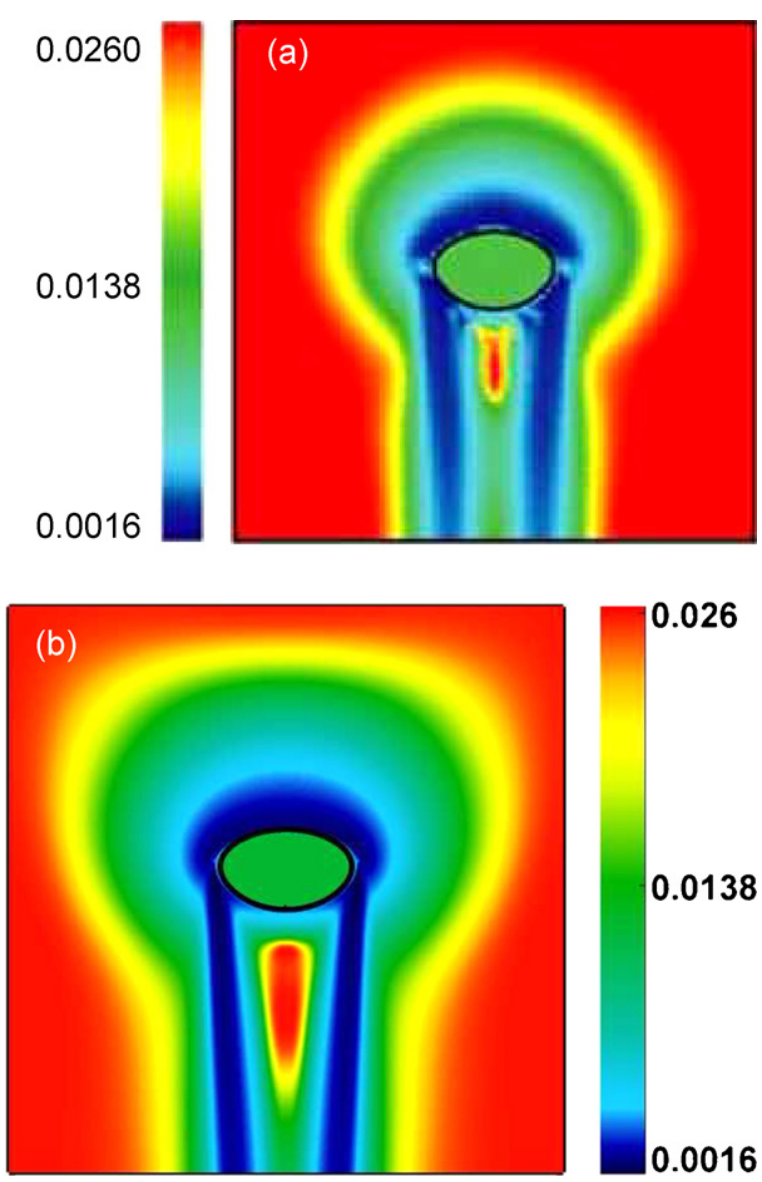

Fig. 11. Comparison of the viscosity distribution and shape of a drop rising in Carreau-Yasuda shear-thinning solution (case 2, $d_{\mathrm{e}}=11.6 \mathrm{~mm}$, viscosity in unit of Pas). (a) VOF ${ }^{[10]}$ and (b) Level set method.

from Table 7 that $R e_{\mathrm{M}}$ values based on the level set method are consistent with those obtained by Ohta et al.

Scrutinizing the VOF results in Figs. 10-12, some flow structures of minor scale are observed around the drop surface. It is some kind of so-called "parasite flows" [22], probably arising from inaccurate account for the interfacial force. However, neat flow structure is obtained from our simulation without any interfacial disturbance. In our method, the "parasitic" surface flow is suppressed by adopting a double fine grid for simulating the motion of drops and bubbles and advancing the deformable interface for each time step starting from the interface instead of the boundary of the computational domain [16].

\section{Concluding remarks}

In this study, the motion of a bubble rising freely through shear-thinning fluids represented by the Carreau model has been experimentally and computationally investigated. An improved level set method is used to simulate the motion of deformable gas bubbles on a staggered Eulerian grid. The numerical method is proved robust and effective for simulating the rise of single air bubbles through a few shear-thinning solutions with the Reynolds number up to 340 . The comparison of simulation of shear-thinning liquids with the present and literature data shows satisfactory agreements for bubble terminal shapes and Reynolds numbers in a wide flow regime.
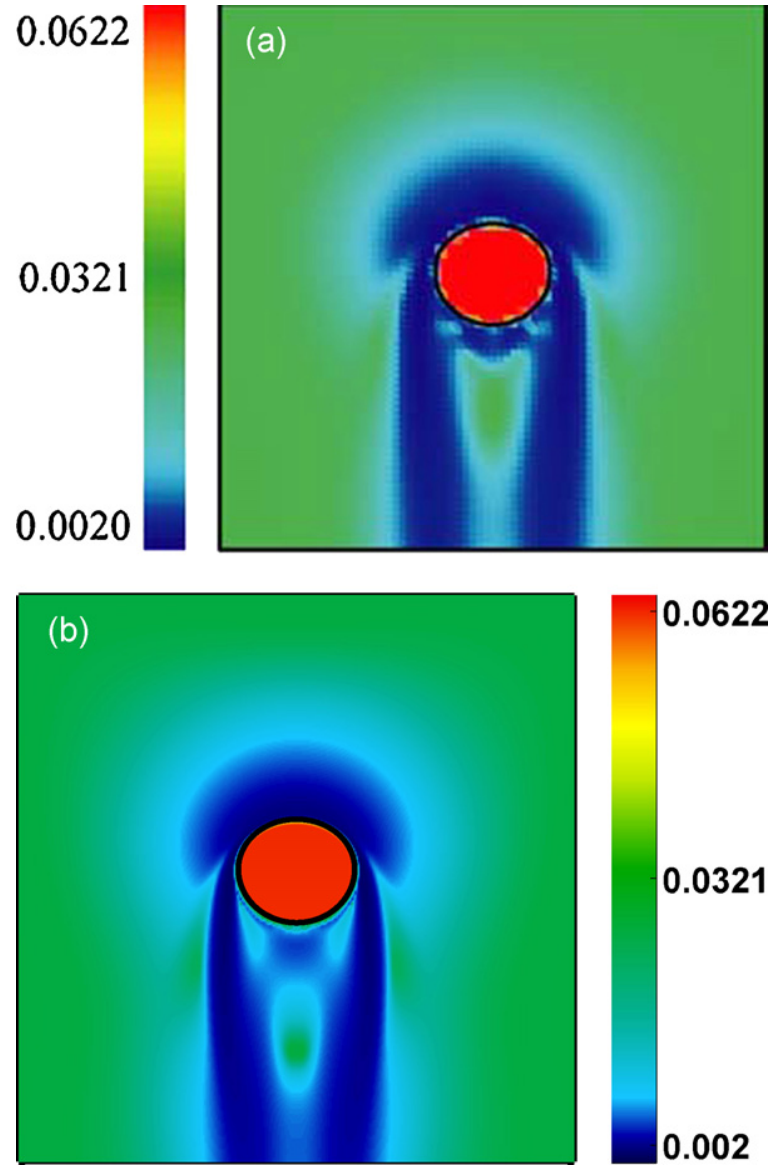

Fig. 12. Comparison of the viscosity distribution and shape of a drop rising in Carreau-Yasuda shear-thinning solution (case $3, d_{\mathrm{e}}=9.8 \mathrm{~mm}$, viscosity in unit of Pas). (a) VOF ${ }^{[10]}$ and (b) Level set method.

For shear-thinning non-Newtonian liquids, the model parameter $n$, the power law index, has considerable influence on the bubble rise motion. The viscosity around the bubble decreases considerably as $n$ becomes small, and as a result, the bubble can rise easily through the shear-thinning liquid and the $R e$ has a larger value than that for Newtonian liquids with the same zero shear-rate viscosity.

The flow structure around a bubble becomes deviated qualitatively from that typical in Newtonian fluids as the shear-thinning property becomes more significant. Behind the bubble a region of wake with high viscosity is observed, leading to the formation of two separated zones in the vortex-shedding regime that may influence the transport of dissolved gas in the wake.

The current computational scheme is expected to provide a sound basis for further investigation on the bubble motion in viscoelastic fluids and the gas-liquid mass transfer in non-Newtonian liquids.

\section{Acknowledgements}

The financial support from the National Natural Science Foundation of China (20990224, 20676134), 973 Program (2010CB630904), the National Project of Scientific and Technical Supporting Program (2008BAF33B03) and the 863 Key Project (2007AA060904) is gratefully acknowledged. 


\section{References}

[1] R.P. Chhabra, Bubbles, Drops, and Particles in Non-Newtonian Fluids, CRC Press, Boca Raton, FL, 1993.

[2] R.P. Chhabra, Bubbles, Drops, and Particles in Non-Newtonian Fluids, second ed., CRC Press, Boca Raton, FL, 2006.

[3] A.A. Kulkarni, J.B. Joshi, Bubble formation and bubble rise velocity in gas-liquid system: A review, Ind. Eng. Chem. Res. 44 (2005) 5873-5931.

[4] W.A. Al-Masry, Effect of scale-up on average shear rates for aerated nonNewtonian liquids in external loop airlift reactors (Communication to the editor), Biotechnol. Bioeng. 62 (1999) 494-498.

[5] N. Kishorea, R.P. Chhabra, V. Eswaranb, Drag on a single fluid sphere translating in power-law liquids at moderate Reynolds numbers, Chem. Eng. Sci. 62 (2007) 2422-2434.

[6] J. Tsamopoulos, Y. Dimakopoulos, N. Chatzidai, G. Karapetsas, M. Pavlidis, Steady bubble rise and deformation in Newtonian and viscoplastic fluids and conditions for bubble entrapment, J. Fluid Mech. 601 (2008) 123-164.

[7] A.J. Wagner, L. Giraud, C.E. Scott, Simulation of a cusped bubble rising in a viscoelastic fluid with a new numerical method, Comput. Phys. Commun. 129 (2000) 227-232.

[8] S.B. Pillapakkam, P. Singh, A level set method for computing solutions to viscoelastic two-phase flow, J. Comput. Phys. 174 (2001) 552-578.

[9] M. Ohta, E. Iwasaki, E. Obata, Y. Yoshida, A numerical study of the motion of a spherical drop rising in shear-thinning fluid system, J. Non-Newtonian Fluid Mech. 113 (2003) 95-111.

[10] M. Ohta, E. Iwasaki, E. Obata, Y. Yoshida, Dynamics processes in a deformed drop rising through shear-thinning fluids, J. Non-Newtonian Fluid Mech. 132 (2005) 100-107.

[11] S. Radl, G. Tryggvason, J. Khinast, Flow and mass transfer of fully resolved bubbles in non-Newtonian fluids, AIChE J. 53 (2007) 1861-1878.
[12] S. Radl, J. Khinast, Prediction of mass transfer coefficients in non-Newtonian fermentation media using first-principles methods, Biotechnol. Bioeng. 97 (2007) 1329-1334.

[13] J.P. Hsu, L.H. Yeh, S.-J. Yeh, Electrophoresis of a rigid sphere in a Carreau fluid normal to a large charged disk, J. Phys. Chem. B 111 (2007) 12351-12361.

[14] S. Osher, J.A. Sethian, Fronts propagating with curvature-dependent speed: algorithms based on Hamilton-Jacobi formulations, J. Comput. Phys. 79 (1988) $12-49$.

[15] M. Sussman, P. Smereka, S. Osher, A level set approach for computing solutions to incompressible two-phase flow, J. Comput. Phys. 114 (1994) 146-159.

[16] C. Yang, Z.-S. Mao, An improved level set approach to the simulation of drop and bubble motion, Chin. J. Chem. Eng. 10 (2002) 263-272.

[17] H. Zhang, L.L. Zheng, V. Prasad, T.Y. Hou, A curvilinear level set formulation for highly deformable free surface problems with application to solidification, Numer. Heat Transfer Part B 34 (1998) 1-20.

[18] J.P. van Doormaal, G.D. Raithby, Enhancements of the SIMPLE method for prediction incompressible fluid flows, Numer. Heat Transfer 7 (1984) 147-163.

[19] D. Bhaga, M.E. Weber, Bubbles in viscous liquid: shapes, wakes and velocities, J, Fluid Mech. 105 (1981) 61-85.

[20] D. Funfschilling, H.Z. Li, Effects of the injection period on the rise velocity and shape of a bubble in a non-Newtonian fluid, Chem. Eng. Res. Des. 84 (2006) 875-883.

[21] J.F. Wang, P. Lu, Z.-H. Wang, C. Yang, Z.-S. Mao, Numerical simulation of unsteady mass transfer by the level set method, Chem. Eng. Sci. 63 (2008) 3141-3151.

[22] J.F. Wang, C. Yang, Z.-S. Mao, Simple weighted integration method for calculating surface tension for suppression of parasitic flow in the level set approach, Chin. J. Chem. Eng. 14 (2006) 740-746. 